\title{
Corrigendum: Fluorophore localization algorithms for super-resolution microscopy
}

\author{
Alex Small \& Shane Stahlheber \\ Nat. Methods 11, 267-279 (2014); published online 27 February 2014; corrected after print 23 July 2014
}

In the version of this article initially published, 3D DAOSTORM was erroneously characterized in Table 2 as using least-squares fits. In fact, 3D DAOSTORM uses an implementation of maximum likelihood estimation (MLE). The error has been corrected in the HTML and PDF versions of the article.

\section{Corrigendum: Evaluation of quantitative miRNA expression platforms in the microRNA quality control (miRQC) study}

Pieter Mestdagh, Nicole Hartmann, Lukas Baeriswyl, Ditte Andreasen, Nathalie Bernard, Caifu Chen, David Cheo, Petula D’Andrade, Mike DeMayo, Lucas Dennis, Stefaan Derveaux, Yun Feng, Stephanie Fulmer-Smentek, Bernhard Gerstmayer, Julia Gouffon, Chris Grimley, Eric Lader, Kathy Y Lee, Shujun Luo, Peter Mouritzen, Aishwarya Narayanan, Sunali Patel, Sabine Peiffer, Silvia Rüberg, Gary Schroth, Dave Schuster, Jonathan M Shaffer, Elliot J Shelton, Scott Silveria, Umberto Ulmanella, Vamsi Veeramachaneni, Frank Staedtler, Thomas Peters, Toumy Guettouche \& Jo Vandesompele Nat. Methods 11, 809-815 (2014); published online 29 June 2014; corrected after print 30 July 2014

In the version of this article initially published, the author Linda Wong was omitted from the author list. The error has been corrected in the HTML and PDF versions of the article.

\section{Corrigendum: A holidic medium for Drosophila melanogaster}

Matthew D W Piper, Eric Blanc, Ricardo Leitão-Gonçalves, Mingyao Yang, Xiaoli He, Nancy J Linford, Matthew P Hoddinott, Corinna Hopfen, George A Soultoukis, Christine Niemeyer, Fiona Kerr, Scott D Pletcher, Carlos Ribeiro \& Linda Partridge Nat. Methods 11, 100-105 (2014); published online 17 November 2013; corrected after print 8 August 2014

In the version of this article initially published, funding information for L.P. was omitted. The author acknowledges funding from the German Federal Ministry of Education and Research (0315893B/Sybacol (Systems Biology of Ageing Cologne)). The error has been corrected in the HTML and PDF versions of the article.

\section{Corrigendum: Independent optical excitation of distinct neural populations}

Nathan C Klapoetke, Yasunobu Murata, Sung Soo Kim, Stefan R Pulver, Amanda Birdsey-Benson, Yong Ku Cho, Tania K Morimoto, Amy S Chuong, Eric J Carpenter, Zhijian Tian, Jun Wang, Yinlong Xie, Zhixiang Yan, Yong Zhang, Brian Y Chow, Barbara Surek, Michael Melkonian, Vivek Jayaraman, Martha Constantine-Paton, Gane Ka-Shu Wong \& Edward S Boyden Nat. Methods 11, 338-346 (2014); published online 9 February 2014; corrected after print 28 August 2014

In the version of this article initially published, the Drosophila transgenic strains were incorrectly reported as generated using Chrimson (Fig. 3, Supplementary Figs. 14-16 and Supplementary Videos 2-6). Owing to a miscommunication, the Drosophila strains were actually generated with CsChrimson, a CsChR-Chrimson chimera replacing the Chrimson $\mathrm{N}$ terminus with the CsChR N terminus. CsChrimson has the same spectral and kinetic properties as Chrimson; these data have been added to the paper in the form of an addendum, and incorrectly listed strains have been fixed in the main text and supplementary materials. The error has been corrected in the HTML and PDF versions of the article. 